\title{
Características de los pacientes diabéticos hospitalizados en dos hospitales de EsSalud Piura
}

Frank Espinoza-Morales ${ }^{1 a}$, Vicente A. Benites-Zapata ${ }^{2 a}$, Kennlly Cardoza-Jiménez ${ }^{3,4 b}$, Marita Quezada-Osoria ${ }^{3,4 b}$, Maryuri Chumbes-Aguirre ${ }^{3,4 b}$.

\section{RESUMEN}

Objetivo: Describir la frecuencia de pacientes con diabetes mellitus tipo 2 (DM2) hospitalizados en Piura.

Material y Métodos: realizamos un estudio transversal en los dos hospitales de mayor nivel de atención de Essalud en Piura: Hospital III José Cayetano Heredia y Hospital II Jorge Reátegui Delgado. Obtuvimos las características clínico-demográficas de las historias clínicas (HC) de los participantes durante el Día Internacional de la Diabetes 2014.

Resultados: De los 183 pacientes hospitalizados en ambos centros, 39 pacientes (21\%) padecían DM2, la media de edad fue $67.8 \pm 11.8$ años. No reportan la glucemia de ingreso y glucemia diaria 17 y 24 participantes, respectivamente. En 31 participantes no se reportó el peso del paciente. El pie diabético fue la principal causa de hospitalización.

Conclusión: La DM2 es muy frecuente en los pacientes hospitalizados de EsSalud Piura, no hay un adecuado registro en las HC de parámetros como peso y control glucémico. (Horiz Med 2015; 15(4): 21-26)

Palabras clave: Pacientes hospitalizados, diabetes mellitus tipo 2, hospitalización. (Fuente: DeCS BIREME).

Characteristics of diabetic patients hospitalized in two Hospitals of EsSalud Piura

ABSTRACT

Objective: To describe the frequency of patients with diabetes mellitus type 2 hospitalized in Piura.

Material and Methods: we conducted a cross-sectional study in two hospitals of greater complexity care of EsSalud in Piura: Hospital III Jose Cayetano Heredia and Hospital II Jorge Reategui Delgado.

Results: We obtained the clinical-demographic characteristics of the participants during the International Diabetes Day 2014. Of the 183 in patients in both centers, 39 patients (21\%) had diabetes mellitus type 2 ; the mean age was $67.8 \pm 11.8$. The medical records do not report hospital admission blood glucose and blood glucose during hospitalization in 17 and 24 participants, respectively. The medical records do not report weight of 31 participants. Diabetic foot was the primary cause of hospitalization.

Conclusion: We conclude that diabetes mellitus type 2 is common in hospitalized patients of Essalud Piura, there is no proper record in the medical records of parameters such as weight and glycemic control. (Horiz Med 2015; 15(4): 21-26)

Key words: Inpatients, diabetes mellitus type 2, hospitalization. (Source: MeSH NLM).

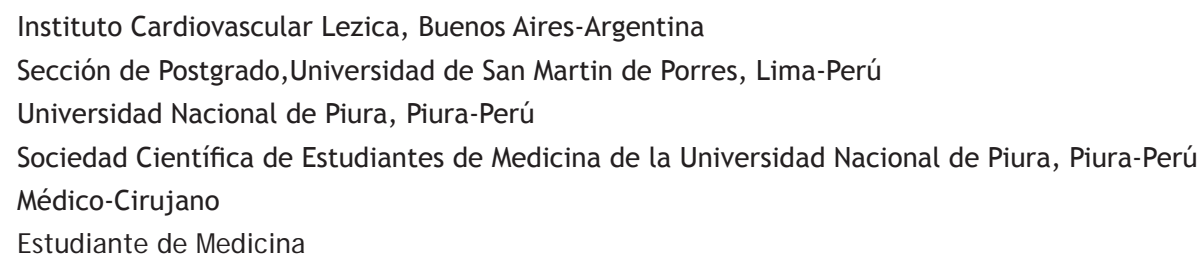




\section{INTRODUCCIÓN}

Actualmente la Diabetes Mellitus (DM) es un problema creciente a nivel global, el número de pacientes diagnosticados con DM se ha duplicado en menos de tres décadas (1).

En Perú, según la Federación Internacional de Diabetes la prevalencia en el 2011 fue 5,4\%, y para el 2030 se proyecta en 6,4\%(2); mientras que el último reporte de la Encuesta Demográfica y de Salud Familiar (ENDES) 2014 se menciona que en nuestro país, el 3,2\% de los mayores de 15 años presentan DM(3).

Estudios demuestran que la presencia de diabetes mellitus tipo 2 (DM2) en pacientes hospitalizados es muy común. La prevalencia de DM2 en pacientes hospitalizados oscila en $5,8 \%$ a $26,2 \%$ y es mucho mayor que la reportada en la población general de los diversos países (4-7).

Incluso estas prevalencias de pacientes DM2 hospitalizados podrían encontrarse infraestimadas ya que a menudo se omite este diagnóstico en las historias clínicas.

Los pacientes hospitalizados con DM2 se diferencian en algunas características de los no diabéticos.

Entre esas características destacan una mayor edad, presencia de enfermedad cardiovascular y una estancia hospitalaria mayor que los pacientes no diabéticos $(4,5,8)$.

Estas características hacen que sean muy susceptibles de un mayor riesgo de desenlaces clínicos desfavorables.

Debido a que no existen datos acerca de pacientes diabéticos hospitalizados en Piura, decidimos llevar a cabo un estudio, cuyo objetivo fue describir las características clínico-demográficas de los pacientes con DM2 hospitalizados en los dos hospitales de mayor nivel de atención de la seguridad social (EsSalud) de Piura.

\section{MATERIAL Y MÉTODOS}

Se llevó a cabo un estudio transversal en los dos hospitales de EsSalud de mayor nivel de complejidad en la región de Piura:

1) Hospital Essalud III José Cayetano Heredia (HJCH) y 2) Hospital EsSalud II Jorge Reátegui Delgado (HJRD).

La población de estudio fueron todos los pacientes con diagnóstico de DM2 que estuvieron hospitalizados en los departamentos de Medicina Interna y Cirugía de ambos hospitales en el Día Internacional de la Diabetes, el 14 de noviembre del 2014.

Excluimos a aquellos participantes que estaban programados para cirugía electiva. La condición de diabético se obtuvo de los registros clínicos de los participantes mirando sus antecedentes médicos y la asistencia por consultorio externo para recoger su medicación antidiabética.

Del mismo modo, se recopilaron variables como edad, sexo, peso, talla, tiempo de diagnóstico de la enfermedad, glucemia de ingreso, glucemia durante la hospitalización, co-morbilidades y si eran usuarios de insulina. La información de las historias clínicas de los participantes incluidos en el estudio fue entregada a los investigadores sin identificadores por la oficina de epidemiologia de ambos centros, de este modo se respetó la confidencialidad de la información de los participantes.

Las variables cuantitativas se informaron como medias y desviación estándar o medianas con rango intercuartílico (RIC) si tenían distribuciones sesgadas.

Las variables categóricas se presentaron con frecuencias y porcentajes. Para las comparaciones entre variables cuantitativas se usó la prueba $t$ de Student o la prueba de Mann-Whitney según correspondía. Para las comparaciones entre variables categóricas se usó la prueba de Chi cuadrado. Para evaluar la correlación entre no presentar glucemia de ingreso y glucemia en hospitalización se usó el coeficiente de Spearman. Los datos fueron procesados con el paquete estadístico STATA 12.1 (StataCorp, TX, USA). 


\section{RESULTADOS}

De un total de 183 pacientes hospitalizados en ambos hospitales evaluados, el $21 \%(n=39)$ tenía DM2. De ellos, 25 participantes procedían del HRCH y 14 participantes del HRJD. En el HRCH la mayoría de participantes $(n=14)$ estaban hospitalizados en el servicio de cirugía mientras que en el HJRD la mayoría de participantes estaba en el servicio de medicina interna $(n=10)$. El promedio de edad fue $67.8 \pm 11.8$, mientras que el $43 \%(n=17)$ fue de sexo masculino y la mediana de tiempo con DM2 fue 9 años RIC (5-20). No hubo diferencias en edad, sexo y tiempo con DM2 entre los participantes de $\mathrm{HRCH}$ y HJRD. Las características clínico-demográficas de los participantes en ambos centros se resumen en la (Tabla 1).

El principal motivo de hospitalización entre los participantes del estudio fue el pie diabético $(n=12)$. Las otras causas de hospitalización fueron déficit motor, fracturas, enfermedad cardiovascular entre otras. En tres participantes no se refería el motivo de hospitalización. Los principales motivos de hospitalización de los pacientes con DM2 se muestran en la (Figura 1).

En 31 de las 39 de historias clínicas de los participantes no se registraron el peso y talla. En relación con el manejo y control de la glucemia, en más de un tercio $(n=17)$ de los participantes no se registró la glucemia de ingreso, mientras que en casi los dos tercios $(n=24)$ de los participantes no se encontraba la glucemia del día. El HRCH presentó la más alta frecuencia de no registrar glucemia de ingreso y durante la hospitalización, 11 y 19 participantes, respectivamente.

La mediana de glucemia de ingreso en los participantes que fue llevada a cabo, fue mayor en los pacientes del HRJD comparada con el HRCH ( $238 \mathrm{mg} / \mathrm{dl}$ versus $121 \mathrm{mg} / \mathrm{dl}$; $\mathrm{p}=0.01$ ), sin embargo no hubo diferencias entre ambos hospitales para la glucemia del día. Asimismo, se encontró que el no presentar glucemia de ingreso está correlacionado positivamente con la falta de glucemia durante la hospitalización (Coeficiente de Spearman =0,48, $\mathrm{p}<$ $0,01)$.

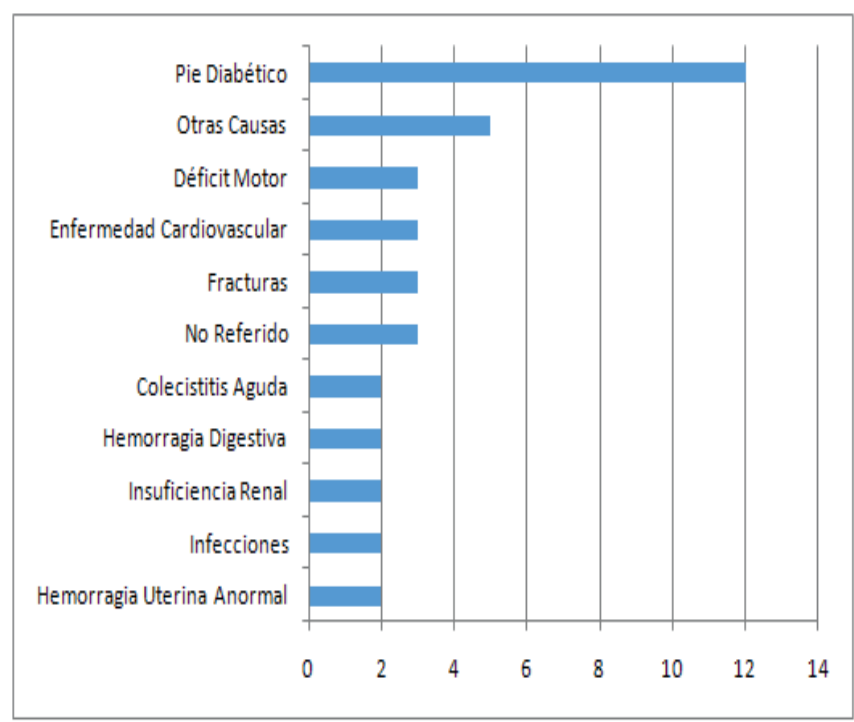

Figura 1. Motivo de hospitalización en los pacientes diabéticos tipo 2 hospitalizados en dos hospitales de Piura en el Día Internacional de la diabetes 2014 .

Tabla 1. Características de los pacientes diabéticos tipo 2 hospitalizados en dos hospitales de Piura en el Día Internacional de la diabetes 2014.

\begin{tabular}{|lcccc|}
\hline \multicolumn{1}{c}{ Variable } & $\begin{array}{c}\text { Total } \\
\mathrm{n}=39\end{array}$ & $\begin{array}{c}\mathrm{HRCH} \\
\mathrm{n}=25\end{array}$ & $\begin{array}{c}\text { HJRD } \\
\mathrm{n}=14\end{array}$ & Valor $\mathrm{p}$ \\
\hline Edad (años) & $67.8 \pm 11.8$ & $67.2 \pm 8.8$ & $69.1 \pm 16.1$ & 0.67 \\
Masculino & $17(43)$ & $10(40)$ & $7(50)$ & 0.55 \\
Tiempo de diagnóstico & $9(5-20)$ & $8(3-18)$ & $18(8-20)$ & 0.20 \\
de diabetes (años) & & & & \\
Glucemia de ingreso (mg/dl)* & $176(115-211)$ & $120(103-195)$ & $238(163-355)$ & 0.01 \\
Glucemia del día (mg/dl)* & $142(104-180)$ & $112(101-162)$ & $150(142-180)$ & 0.28 \\
Hipertensión & $23(59)$ & $15(60)$ & $8(57)$ & 0.86 \\
Tratamiento con insulina & $17(44)$ & $5(20)$ & $1286)$ & $<0.01$ \\
& & & & \\
\hline
\end{tabular}

*Sólo los participantes que tenían registrada la glicemia.

Media \pm desviación estándar,mediana (rango intercuartílico). Número (porcentaje). 


\section{DISCUSIÓN}

En nuestro estudio encontramos que la quinta parte de los pacientes hospitalizados padecían diabetes mellitus tipo 2. A su vez una considerable cantidad de estos pacientes con DM2 no tenían glucemia de ingreso y posteriormente una mayor cantidad de ellos no tenían glucemia durante la hospitalización.

La prevalencia de diabetes en Perú según la ENDES 2014 fue 3.2\% para los mayores de 15 años, sin embargo esta cifra es una infraestimación de la real prevalencia dado que el diagnóstico de DM es recolectado en ENDES mediante autoreporte (3).

De ese modo, una estimación hecha en otro estudio para el año 2010 evidencia una prevalencia de $5.6 \%$ de adultos con DM en Perú aj ustada por la población nacional (9).

Para la ciudad de Piura existen algunos estudios llevados a cabo en algunos grupos poblacionales que muestran prevalencias de $5 \%$ a $6.7 \%(10,11)$. Aunque poco precisos, estas prevalencia estimadas evidencian a Piura como una ciudad con una presencia importante de pacientes con DM.

La frecuencia de pacientes hospitalizados con DM2 encontrada en nuestro estudio fue similar a lo reportado en Australia y Barbados $(4,8)$, pero mayor a lo reportado en Reino Unido y Japón $(6,7)$.

En Perú no pudimos encontrar un estudio que reporte el porcentaje de pacientes hospitalizados con DM2 para compararlo con nuestros hallazgos.

Dadas las cifras estimadas de DM2 para la población general; la presencia de un paciente con DM2 por cada cinco pacientes hospitalizados evidencia una frecuencia muy elevada de DM2 en los dos hospitales de mayor complejidad de la seguridad social de Piura.

Estas cifras encontradas son preocupantes ya que la hospitalización de pacientes diabéticos se ha asociado a mayores complicaciones $(12,13)$, con un mayor tiempo de estancia hospitalaria y con un incremento en los costos de atención debido a las complicaciones(14).
Siendo EsSalud una institución administradora de fondo de aseguramiento en salud que tiene un plan de beneficios denominado plan específico de salud con cobertura de todas las necesidades de salud (15), el uso eficiente de los recursos económicos es vital para garantizar su sostenibilidad en el tiempo.

De todas las causas de hospitalización encontradas en pacientes con DM2 en nuestro estudio, el pie diabético fue la principal, muy similar a lo reportado por Taylor et al (8). En el tratamiento de esta complicación son necesarias estrategias de abordaje terapéutico debido a que el paciente diabético, tiene 25 veces más riesgo de amputación que una persona normal (16).

Por lo tanto, un adecuado control glucémico es necesario para un óptimo manejo de pacientes DM2 hospitalizados. Sin embargo, en nuestro estudio, una gran cantidad de pacientes no tenían control de glucemia al ingreso al hospital.

Asimismo, la falta de glucemia de ingreso se relacionó con la falta de glucemia en la hospitalización.

Esto es preocupante, porque tanto la hipoglucemia y la hiperglucemia se han asociado con mayor tiempo de hospitalización, peores desenlaces clínicos, mayor riesgo de complicaciones y mortalidad $(7,17)$.

Otro hallazgo interesante fue que un alto porcentaje de historias clínicas no registró peso ni talla de los pacientes, lo cual indica que estos parámetros no son considerados durante la evaluación de los pacientes hospitalizados.

El peso y la talla son importantes para la atención clínica ya que son utilizados regularmente por el médico para calcular el volumen de hidratación, las dosis de medicación de acuerdo con la superficie del cuerpo y mantener el equilibrio electrolítico adecuado.

Nuestro estudio tiene las limitaciones características de un estudio transversal, además de una limitación adicional debido a la utilización de las historias clínicas en la investigación. Sin embargo, nos da 
una visión general del problema y nos permite abrir campo de la investigación sobre el tema.

En conclusión, existen una gran cantidad de pacientes hospitalizados que padecen DM2 y una buena parte de ellos no cuentan con glucemia de ingreso ni de hospitalización.

Ante lo cual, sugerimos futuras investigaciones para valorar los desenlaces clínicos en los pacientes con DM2 hospitalizados y conocer sus principales factores de riesgo. Además, es necesario realizar auditorías de las historias clínicas en cuanto a la calidad del registro y de la atención, para implementar las intervenciones necesarias para mejorar el manejo clínico de estos pacientes.
Fuentes de financiamiento

Este estudio ha sido financiado por los autores.

\section{Conflicto de interés}

Los autores declaran no tener ningún conflicto de interés.

\section{Correspondencia:}

Vicente A Benites-Zapata

Dirección: Sección de Postgrado, Universidad de San Martin de Porres (USMP), Av. Alameda del Corregidor $\mathrm{N}^{\circ}$ 1531, La Molina, Lima, Perú.

Teléfono: 995523081

Correo electrónico: vbeniteszapata@gmail.com 


\section{REFERENCIAS BIBLIOGRÁFICAS}

1. Danaei G, Finucane MM, Lu Y, Singh GM, Cowan MJ, Paciorek CJ, et al., others. National, regional, and global trends in fasting plasma glucose and diabetes prevalence since 1980: systematic analysis of health examination surveys and epidemiological studies with 370 country-years and $2 \cdot 7$ million participants. The Lancet. 2011;378(9785):31-40.

2. Atlas ID. 6th edn. Int Diabetes Fed Belg. 2013;160.

3. Instituto Nacional de Estadística e Informática. Perú: enfermedades no transmisibles y transmisibles, 2014. Instituto Nacional de Estadística e Informática; 2015.

4. Bach LA, Ekinci El, Engler D, Gilfillan C, Hamblin PS, Maclsaac RJ, et al. The high burden of inpatient diabetes mellitus: the Melbourne Public Hospitals Diabetes Inpatient Audit. Med J Aust. 2014;201(6):334-8.

5. Rayman G. National Health Service: National Diabetes Inpatient Audit 2012. U K. 2013;

6. Kubo T, Fujino $\mathrm{Y}$, Murata A, Ichimiya $\mathrm{Y}$, Kuwabara K, Fujimori $\mathrm{K}$, et al. Prevalence of type 2 diabetes among acute inpatients and its impact on length of hospital stay in Japan. Intern Med. 2011;50(5):405-11.

7. Umpierrez GE, Isaacs SD, Bazargan N, You X, Thaler LM, Kitabch AE. Hyperglycemia: an independent marker of in-hospital mortality in patients with undiagnosed diabetes. J Clin Endocrinol Metab. 2002;87(3):978-82.

8. Taylor CG, Krimholtz M, Belgrave KC, Hambleton I, George $\mathrm{CN}$, Rayman G. The extensive inpatient burden of diabetes and diabetes-related foot disease in Barbados. Clin Med. 2014;14(4):367-70.

9. Shaw JE, Sicree RA, Zimmet PZ. Global estimates of the prevalence of diabetes for 2010 and 2030. Diabetes Res Clin Pract. 2010 Jan 1;87(1):4-14.
10. Seclen Santisteban S, Leey Casella J, Villena Pacheco EA, Herrera Mandelli BR, Menacho J, Carrasco A, et al. Prevalencia de Obesidad, Diabetes Mellitus, Hipertensión Arterial e Hipocolesterolemia como Factores de Riesgo Coronario y Cerebrovascular en Población Adulta de la Costa, Sierra y Selva del Perú. Acta Méd Peru. 1999 Sep;17(1):8-12.

11. Seclén Santisteban S. Aspectos epidemiológicos y genéticos de la diabetes mellitus en la población peruana. Rev Medica Hered [Internet]. 2013 [cited 2015 Sep 30];7(4). Available from: http:// www.upch.edu.pe/vrinve/dugic/revistas/index.php/RMH/ article/download/508/475

12. Osuna M, Rivera MC, Bocanegra $\mathrm{C}$ de $\mathrm{J}$, Lancheros $\mathrm{A}$, Tovar $\mathrm{H}$, Hernández Jl, et al. Caracterización de la diabetes mellitus tipo 2 y el control metabólico en el paciente hospitalizado. Acta Médica Colomb. 2014;39(4):345.

13. Membreño Mann JP, Zonana Nacach A. Hospitalización de pacientes con diabetes mellitus. Causas, complicaciones y mortalidad. Rev Med IMSS. 2005;43(2):97-101.

14. Mata M, Antoñanzas F, Tafalla M, Sanz P. El coste de la diabetes tipo 2 en España: El estudio CODE-2. Gac Sanit. 2002 Dec;16(6):511-20.

15. Alcalde-Rabanal JE, Lazo-González O, Nigenda G. Sistema de salud de Perú. Salud Pública México. 2011;53:s243-54.

16. Bakker K, Apelqvist J, Lipsky BA, Van Netten JJ, Schaper NC, International Working Group on the Diabetic Foot (IWGDF). The 2015 IWGDF guidance documents on prevention and management of foot problems in diabetes: development of an evidence-based global consensus. Diabetes Metab Res Rev. 2015 Sep 27;

17. Hsu P-F, Sung S-H, Cheng H-M, Yeh J-S, Liu W-L, Chan W-L, et al. Association of clinical symptomatic hypoglycemia with cardiovascular events and total mortality in type 2 diabetes a nationwide population-based study. Diabetes Care. 2013;36(4):894 900. 